\title{
Space-Time Digital Filtering of Radio Astronomical Signals using 3-D Cone Filters
}

\author{
Najith W. Liyanage \\ Department of Electrical and Computer Engineering, \\ University of Victoria, Victoria, BC, Canada \\ E-mail: najith@ece.uvic.ca \\ Leonard T. Bruton \\ Department of Electrical and Computer Engineering, \\ University of Calgary, Calgary, AB, Canada \\ E-mail: brutoneucalgary.ca

\section{Pan Agathoklis* and Chamira Edussooriya} \\ Department of Electrical and Computer Engineering, \\ University of Victoria, Victoria, BC, Canada \\ E-mail: pandece.uvic.ca, chamira@ece.uvic.ca
}

In this paper, 3-D space-time (ST) processing is proposed as a technique to enhance signals of interest (SOIs) by attenuating near-over-the-horizon radio frequency interferences (RFIs) that exist on dense phased arrays used in the next generation of radio telescopes. The proposed approach is based on the difference between the regions of support (ROSs) in the 3-D frequency domain of the Fourier transforms of the SOIs and RFIs. A 3-D linear phase filter bank structure, consisting of 1-D FIR filters and 2-D circularly symmetric FIR filters, is employed to suppress the RFIs. The performance of the proposed 3-D ST filtering approach is illustrated using synthesized broadband (BB) SOIs and BB RFIs on dense aperture arrays (DAAs) and on focal plane arrays (FPAs). Results are presented that indicate the potential for successful mitigation of near-over-the-horizon BB RFIs without significantly distorting the BB SOIs. The advantage of the proposed ST filtering approach is its inherent capability of BB processing.

RFI mitigation workshop

29-31 March 2010

Groningen, the Netherlands

\footnotetext{
${ }^{*}$ Speaker.
} 


\section{Introduction}

The next generation of radio telescopes requires unprecedented levels of sensitivity, resolution, and field of view (FOV) that cannot be achieved with current radio telescopes. Radio astronomers and scientist are working on the development of next generation telescope designs, such as the square kilometre array (SKA) [1]. Depending on the observations, the signals of interest (SOIs) in the next generation of radio telescopes, such as the SKA, may span at least a decade of temporal frequency and are therefore broadband (BB). Moreover, the requirement of unprecedented levels of sensitivity makes radio frequency interference (RFI) a major problem. In achieving the science specifications, two emerging synthetic aperture applications of dense phased arrays are being considered [2], [3] for the so-called mid-frequency range from about 0.3-2 GHz: they are, rectangular dense aperture arrays (DAAs) (as in Fig. 1(a)) and/or focal plane arrays (FPAs) in the focal plane of paraboloidal dishes (as in Fig. 1(b)).

Previously reported works on the mitigation of RFIs are based on temporal blanking techniques which are mostly suitable for intermittent RFI [2], on spatial filtering techniques which use subspace-projection methods [3]-[5], on cancellation techniques which use a reference signal approach [6], and on post-correlation cancellation and anti-coincidence techniques [7]. Such methods are mostly being investigated in the context of narrowband applications. However, for next generation synthetic aperture systems, such as the SKA, the SOIs are inherently BB and therefore it is an interesting possibility to consider a suitable BB approach capable for attenuating (i.e., mitigating) RFI signals without significantly distorting BB SOIs.

Three-dimensional (3-D) space-time (ST) filtering for processing BB space-time signals has been applied in many fields, such as wireless communications, video processing, radar, sonar, seismic signal processing, etc. [8]. Previous studies [8]-[13] (and many references to be found there) confirm the utility of these methods, based on the enhancement of 3-D ST plane waves (PWs) based on their direction of arrivals (DOAs) or so-called linear trajectory (LT) 3-D SOIs, based on their trajectories. The 3-D stopbands attenuate undesirable 3-D signals, such as noise, interference, multipath signals, etc. These methods are inherently capable of processing BB SOIs in the 3-D filter passbands.

In this paper, we describe the application of 3-D space-time cone filters for faithfully transmitting passband BB SOIs while significantly attenuating near-over-the-horizon BB RFI stopband signals on dense phased arrays for radio astronomy applications. The phased arrays include both the FPAs and the DAAs that are emerging for next generation radio astronomical applications. BB near-over-the-horizon RFI signals are modeled using ST PW signals that propagate at, or close to, light speed $c$ with 3-D spatial DOAs that are on (or sufficiently close to) the plane of the phased array. Such ST PW signals have 3-D ST line spectra in $\left(\omega_{x}, \omega_{y}, \omega_{c t}\right) \in \mathbb{R}^{3}$, where $\omega_{c t}=\omega_{t} / c \in \mathbb{R}$, having regions of support (ROSs) (ROS is the region of the 3-D frequency space where the Fourier spectrum of the signal is nonzero.) that are on, or close, to the surface of the 3-D spectral light cone $\left(\omega_{x}^{2}+\omega_{y}^{2}=\omega_{c t}^{2}\right)$ [8]. These BB ST models indicate that BB RFI signals can be attenuated by means of a suitable 3-D ST cone filter encompassing the ROSs of the BB SOIs in the 3-D passband and the region close to the spectral light cone in the 3-D stopband. The subsequent beamforming/correlation operations demand that the ST filtering preserve the phase information of the SOIs. Further, it is required that the SOIs undergo minimal distortion while providing attenuation of the 
RFIs, implying a 3-D linear phase transfer function.

In previous work [9]-[12], the axis of the passband of cone filer banks may be oriented to point at the DOA. However, for radio astronomy applications having the requirement that the passband be circularly symmetric around the temporal frequency axis, a relatively simple 3-D cone filter bank architecture is described (first proposed in [14] and consisting of 1-D FIR filters and 2-D circularly symmetric FIR filters).

This paper is organized as follows: in section 2, the modeling of the RFIs, SOIs and their ROSs in the 3-D frequency domain are discussed and, in section 3, the 3-D cone filter is presented. Finally, in section 4 the performance of the proposed filtering approach is illustrated using synthesized BB SOIs and BB RFIs on DAAs and FPAs.

\section{Near-Over-the-Horizon Radio Frequency Interferences (RFIs) and Signals of Interest (SOIs)}

The type of RFI considered here are near-over-the-horizon artificial RFIs, often scattered by the intervening terrain, that can be modeled as BB ST PWs arriving at the antenna array from a direction of arrival (DOA) that does not fall within the range of the DOAs of the SOIs. A BB ST PW signal, incident on a co-planar array with a spatial DOA of $\left(\theta_{p w}, \phi_{p w}\right)$ has its ROS in the 3-D spatio-temporal frequency domain on a straight line through the origin with an orientation of $\left(\alpha_{p w}, \beta_{p w}\right)=\left(\tan ^{-1}\left(\sin \theta_{p w}\right), \phi_{p w}\right)$ in $\left(\omega_{x}, \omega_{y}, \omega_{c t}\right)$, where $\alpha_{p w}$ and $\beta_{p w}$ are the angles between ROS and $\omega_{c t}$ axis, and $\omega_{x}$ axis and projection of ROS onto the $\omega_{x} \omega_{y}$ plane, respectively.

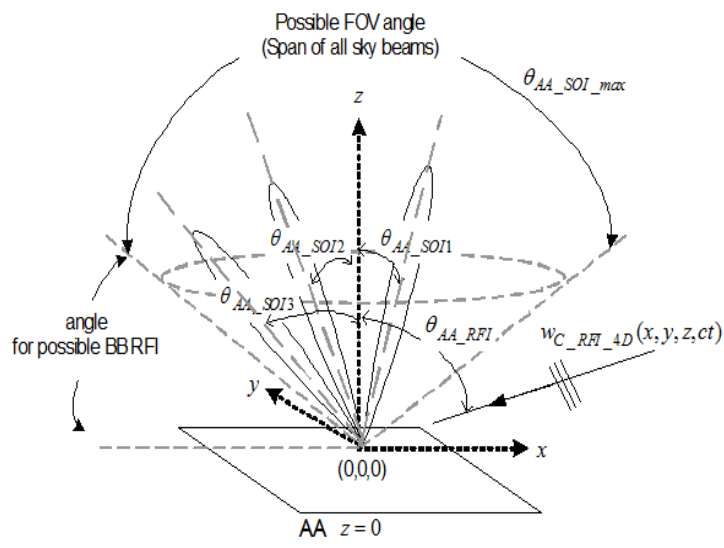

(a)

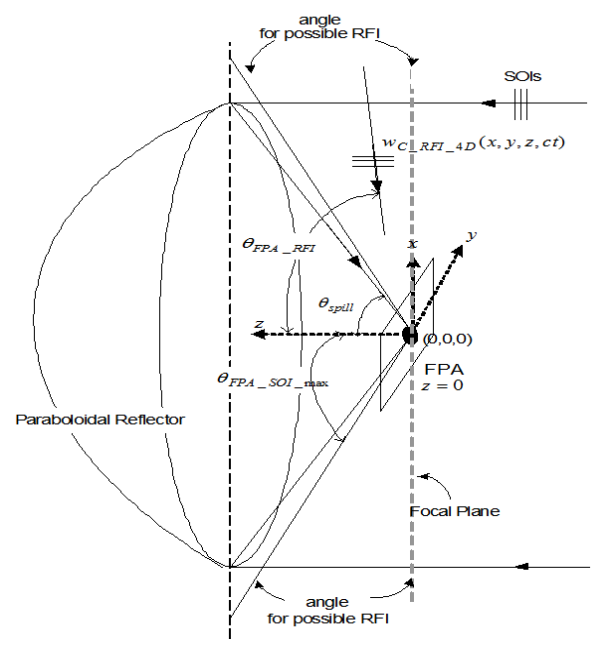

(b)

Figure 1: ST PWs as SOI and RFI signals distinguished by their spatial Direction of Arrival (DOA) (a) on dense aperture arrays (DAAs) (b) on focal plane arrays (FPAs).

The following scalar analysis is limited to the single polarization of EM PWs but may be separately applied to the second polarization. In the case of FPAs, the angle subjected to possible RFI intrusion corresponds to the angle between the focal plane and the physical spill over angle, $\theta_{\text {spill }}$ as illustrated in Fig. 1(b). Due to the diffraction of SOIs in the outer-rim of the paraboloidal 


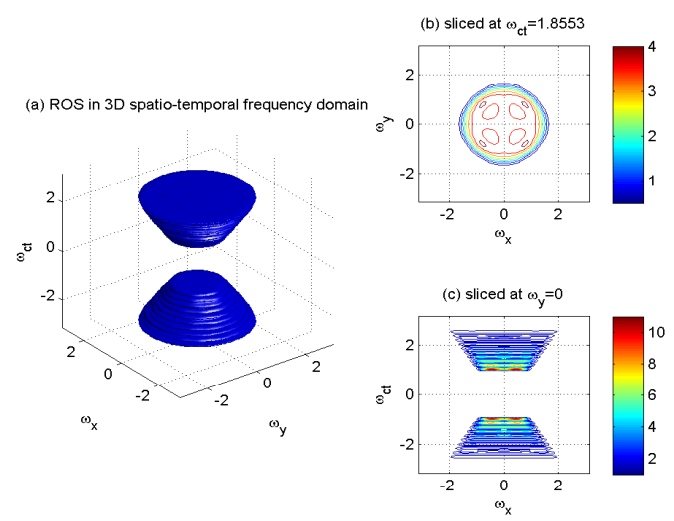

Figure 2: The magnitude spectrum of an SOI on the FPA due to a broadside broadband (0.7-1.8 $\mathrm{GHz}$ ) ST PW incident on a paraboloidal reflector of diameter $D=15 \mathrm{~m}$ and focal-ratio $f / D=0.5$; (a) 3-D ROS containing 97\% energy (b) a slice at $\omega_{c t}=1.8553$ (c) a slice at $\omega_{y}=0$ [16].

dish, caused by non-zero taper at the edge of the outer-rim and the considerable size of the FPA, $\theta_{\max }$ on the FPA may exceed $\theta_{\text {spill }}$.

In the case of DAAs, any BB ST PW that is incident on the array, and having a DOA corresponding to an angle inside the FOV area (i.e., $0 \leq \theta_{p w} \leq \theta_{\max }$ ) shown in Fig. 1(a), is considered to be a BB ST SOI. The respective ROSs of the 3-D spectra of SOIs on DAAs are close to straight lines through the origin in $\left(\omega_{x}, \omega_{y}, \omega_{c t}\right)$.

In the case of FPAs (and in contrast to DAAs), it has been proposed and confirmed [15] by Gunaratne and Bruton that dish-reflected signals (including all celestial SOIs from all DOAs) form a diffraction field that, in the focal plane and close to the focal point, has a 3-D ROS in $\left(\omega_{x}, \omega_{y}, \omega_{c t}\right)$ that is very close to a wide-angle cone having axis $\omega_{c t}=0$ and cone vertex angle given by $\theta_{\max }$. Fig. 2 illustrates the magnitude spectrum of an SOI on the FPA due to a broadside broadband (0.7-1.8 GHz) ST PW incident on a paraboloidal reflector of diameter $D=15 \mathrm{~m}$ and focal-ratio $f / D=0.5$.

\section{3-D Cone Filter Design}

We now describe a suitable 3-D cone filter bank for processing the ST uniformly-sampled and digitized 3-D FPA diffraction field, having the the required linear phase circularly-symmetric 3-D passband that encloses the ROSs of the SOIs such that the 3-D stopband attenuates the ROSs of near-over-the-horizon RFIs. Obviously, steep 3-D transitions between ideally-flat passband and good stopband attenuation are desirable.

The 3-D ST filter bank structure proposed in [14] is used here. This 3-D linear phase FIR cone filter is based on a filter bank structure consisting of 1-D linear phase FIR filters and 2-D circularly symmetric zero phase FIR filters, yielding the linear phase 3-D frequency response transfer function shown in Fig. 3 and Fig. 4(c).

\section{Simulated Verification of Performance}

We describe an example of two BB RFI signals having spatial DOAs given by $\left(\theta_{1}, \phi_{1}\right)=$ 


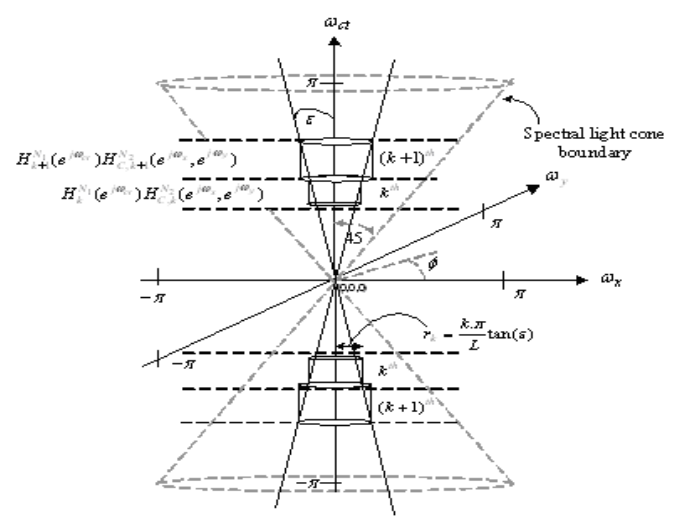

Figure 3: The linear phase analytic cone filter approximation with L bands.

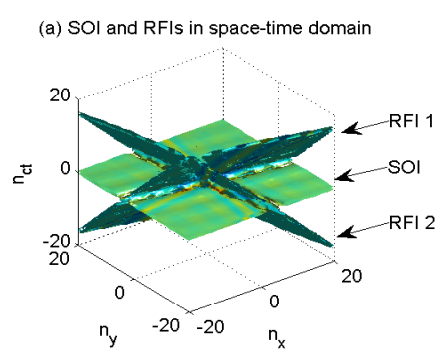

(c) $3 \mathrm{~dB}$ surface of the approximated cone filter

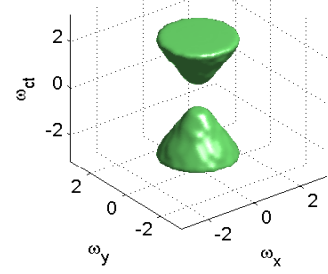

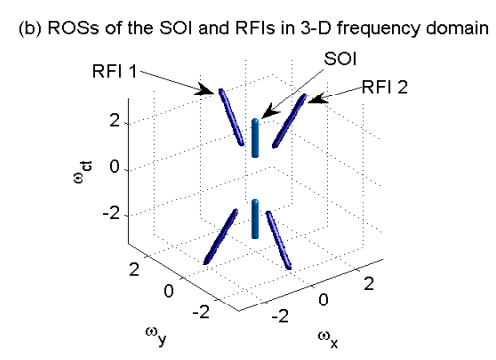

(d) ROS of the output in 3-D frequency domain

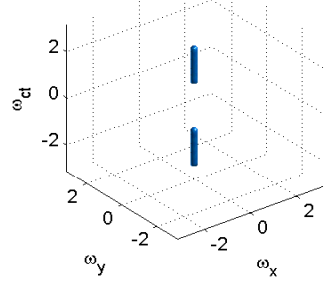

Figure 4: Illustration of the 3-D cone filtering concept for mitigation of RFIs (a) SOI and RFIs in ST domain (b) ROSs of SOI and RFIs in 3-D frequency domain (c) $3 \mathrm{~dB}$ surface of the cone filter with $\varepsilon=30^{\circ}$ (order of $40 \times 40 \times 40$ ) (d) ROS of the output signal in 3-D frequency domain[16].

$\left(85^{0}, 80^{\circ}\right)$ (RFI 1$)$ and $\left(\theta_{2}, \phi_{2}\right)=\left(90^{0}, 10^{\circ}\right)($ RFI 2). The SOI is also a BB PW having spatial DOA given by $\left(\theta_{0}, \phi_{0}\right)=\left(0^{0}, 0^{0}\right)$ (SOI). The BB property of these SOIs is ensured by synthesizing them from multiple superimposed equal-amplitude cosine functions having monochromatic frequencies that densely cover the desired mid-frequency range $0.7-1.8 \mathrm{GHz}$.

The elements of the DAA are assumed to be rectangularly-distributed, uniformly-weighted and spaced to satisfy the Nyquist condition. The SOI has been filtered using the 3-D cone filter bank with results as summarized in Fig. 4. In Fig. 4(a), we show the SOI along with two RFI signals received by an array of $\left(N_{x} \times N_{y}\right)=(41 \times 41)$ in ST domain while in Fig. 4(b), the ROSs of three signals in 3-D frequency domain are shown. In Fig. 4(c), the $3 \mathrm{~dB}$ surface of the frequency response of the 3-D cone filter of order $40 \times 40 \times 40$ and $\varepsilon=30^{0}$ is shown, and in Fig. 4(d), the output of the filter in the 3-D frequency domain is shown. It is observed in Fig. 4(d) that the two RFI signals have been significantly attenuated, as required. 
The improvement of the signal-to-interference ratio (SIR) for various cone angles has been obtained. The RFIs are the same signals as before. And, the SOI in the case of DAA is the same as before while in the case of FPA the SOI has a spectrum with ROS in a cone as shown in Fig. 2. The FPA is assumed to be comprised of 225-elements, $\left(N_{x} \times N_{y}\right)=(15 \times 15)$, and the order of the 3 -D cone filter was chosen as $14 \times 14 \times 40$. In both cases, the energy of the SOI is 100 times lower than the energy of each of the RFIs, representing a very weak SOI. The SIR of the input and output signals for various cone angles for both DAA and FPA are shown in Fig. 5(a) and Fig. 5(b), respectively.

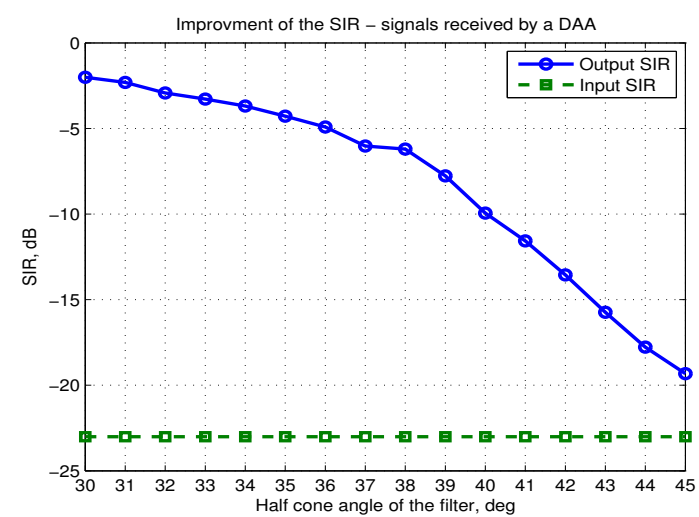

(a)

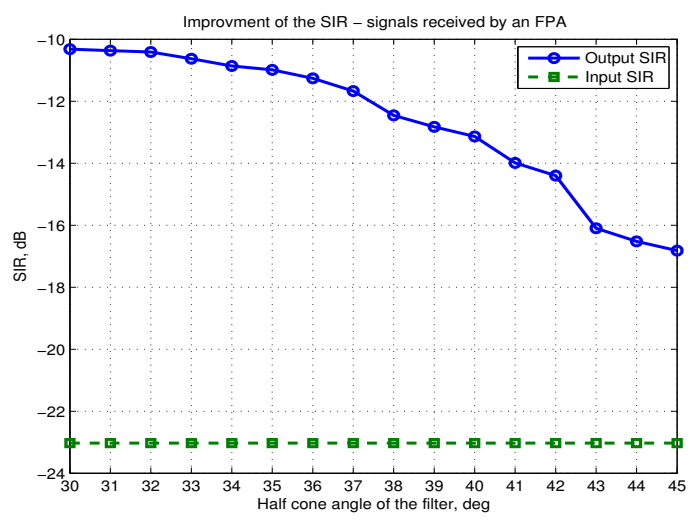

(b)

Figure 5: Improvement of SIR (a) DAA of $\left(N_{x} \times N_{y}\right)=(41 \times 41)$ (b) FPA of $\left(N_{x} \times N_{y}\right)=(15 \times 15)$.

It is observed that the improvement in SIR is reduced when the cone angle is increased. This is due to the fact that, for increased angle, more of the energy of the RFI encroaches into the 3-D passband. Further the improvement in SIR is significantly higher for DAAs than for FPAs. This is due to the fact that the spectral energy of the SOI is concentrated closer to the temporal frequency axis in the case of DAAs relative to FPAs.

\section{Conclusions}

A 3-D ST filtering approach is described for attenuating near-over-the-horizon BB RFI signals in DAAs and FPAs for potential application in next generation radio astronomy applications. For sufficiently large arrays, the results indicate significant attenuation of near-over-the-horizon RFI signals. The length of the proposed 3-D FIR cone filter in each spatial direction cannot exceed the corresponding number of spatial samples: for example: $15 \times 15$ for the 225 -element FPA reported in Fig. 5(b). This maximum length limits the improvement in SIR that can be achieved and, in particular, the maximum stop band attenuation and the accuracy of the passband inside of the cone.

\section{Acknowledgment}

The authors thank to Dr. Bruce Veidt and Dr. Rick Smegal, Dominion Radio Astrophysical Observatory, Penticton, BC, for helpful discussions and directing us to various references. 
This work has been financially supported by Natural Sciences and Engineering Research Council, Canada.

\section{References}

[1] P. J. Hall (Ed.), The Square Kilometre Array: An Engineering Perspective, Springer, 2005.

[2] S. W. Ellingson and G. A. Hampson, Mitigation of radar interference in L-band radio astronomy, ApJS, 147, 167-176, 2003.

[3] J. Raza, A. -J. Boonstra, and A. -J. van der Veen, Spatial filtering of RF interference in radio astronomy, IEEE Sig. Proces. Letters, 9, 64-67, 2002.

[4] A. Leshem, A. -J. van der Veen, and A. Boonstra, Multichannel interference mitigation techniques in radio astronomy, ApJS, 131, 355-373, 2000.

[5] B. D. Van Veen and K. M. Buckley, Beamforming: a versatile approach to spatial filtering, IEEE ASSP Mag., 5, 4-24, 1988.

[6] B. D. Jeffs, L. Li, and K. F. Warnick, Auxiliary antenna-assisted interference mitigation for radio astronomy arrays, IEEE Trans. Sig. Proces., 53, 439-451, 2005.

[7] F. Briggs, J. Bell, and J. Kesteven, Removing radio interference from contaminated astronomical spectra using an independent reference signal and closure relations, Astronom. J., 120, 3351-3361, 2000.

[8] D. E. Dudgeon and R. M. Mersereau, Multidimensional Digital Signal Processing, Englewood Cliffs NJ, Prentice-Hall Inc, 1984.

[9] L. T. Bruton, Selective filtering of spatio-temporal plane waves using 3D cone filter banks, Comm., Computers and Sig. Proces., IEEE Pacific Rim Conference, 1, 67-70, 2001.

[10] L. T. Bruton and S. Singh, Plane wave filtering using a novel $3 D$ cone-stop filter bank, The 45th Midwest Symposium on CAS, 3, 676-679, 2002.

[11] L. T. Bruton, Three-dimensional cone filter banks, IEEE Trans. CAS, 50, 208-216, 2003.

[12] B. Kuenzle and L. T. Bruton, 3-D IIR filtering using decimated DFT-polyphase filter bank structures, IEEE Trans. CAS, 53, 394-408, 2006.

[13] M. Bolle, A closed form design method for recursive 3-D cone filters, IEEE Int. Conf. on Acoustics, Speech, and Sig. Proces., 6, 141-144, 1994.

[14] N. Liyanage, L. Bruton, and P. Agathoklis, On the attenuation of interference and mutual coupling in antenna arrays using 3D space-time filters, IEEE Pacific Rim Conference, 146-151, 2009.

[15] T. K. Gunaratne and L. T. Bruton, UC-FPFC: A MATLAB program to evaluate the electric field on the observation plane of a prime focus paraboloidal reflector, Internal Report, University of Calgary, 2008.

[16] N. Liyanage, 3D space-time digital filtering for radio astronomy, Master's thesis, Dept. of Electrical and Computer Eng., University of Victoria, BC, Canada, 2009. 